\title{
Loss of AMIGO2 causes dramatic damage to cardiac preservation after ischemic injury
}

\author{
Xuhui $\mathrm{Ma}^{1}$, Pengfei $\mathrm{Hu}^{2}$, Haifeng Chen ${ }^{3}$, Tianfu Fang ${ }^{1}$ \\ ${ }^{1}$ Department of Cardiology, Xixi Hospital of Hangzhou, Hangzhou, Zhejiang, China \\ ${ }^{2}$ Department of Cardiology, The Second Affiliated Hospital of Zhejiang Chinese \\ Medical University, Hangzhou, Zhejiang, China
}

${ }^{3}$ Nursing Department, Community Health Service Center of Yuanpu, Hangzhou, Zhejiang, China

\begin{abstract}
Background: Recent studies have identified amphoterin-induced gene and open reading frame (AMIGO2). The role of AMIGO2 in tumour research is well-studied, but its role in ischemic heart diseases is seldom reported. In the present study, the role of AMIGO2 in myocardial infarction (MI) is under investigation for the first time.

Methods: For in vitro studies, cardiomyocytes (CMs) and endothelial cells (ECs) were isolated from both AMIGO2 knockout (KO) and WT mice. The apoptosis of CMs was tested after $48 \mathrm{~h}$ of ischemic stimulation. A proliferation test was implemented after 7 days of normoxic incubation and tube formation on ECs. For in vivo studies, the MI model was built in mice hearts. Echocardiographic evaluation was performed at 3 days and 28 days post-MI, while the hemodynamics test was performed at 28 days post-MI. The histological results of the apoptosis, proliferation, angiogenesis and infarct zone assessments were determined using terminal deoxynucleotidyl transferase-mediated dUTP nick end-labelling (TUNEL) assay, Ki67 staining, $\alpha-S M A / C D 31$ immunostain and the Masson-Trichrome method, respectively. The expression changes of the Akt pathway and related proteins were confirmed using both quantitative real-time polymerase chain reaction ( $q R T-P C R)$ and Western blot.

Results: The present results demonstrated that AMIGO2 deficiency caused more CMs suffering apoptosis, lower proliferation and less angiogenesis in vitro and in vivo. Weaker cardiac function and larger scar formation were detected in AMIGO2 KO mice, and increased expression of active-caspase-3 and decreased expression of PDK1, p-Akt, Bcl-2/Bax and VEGF occurred.

Conclusions: Herein the findings indicate that AMIGO2 deficiency plays an attenuated cardio-protective role in ischemic heart disease via inactivation of the PDK1/Pten/Akt pathway. (Cardiol J 2019; 26, 4: 394-404)
\end{abstract}

Key words: AMIG02, myocardial infarction, apoptosis, angiogenesis

\section{Introduction}

Myocardial infarction (MI) is the world's leading cause of morbidity and mortality, and ischemic injury induces apoptotic loss of the myocardium $[1,2]$, which leads to ventricular remodeling $[3,4]$. Avoiding the myocardium loss and inhibiting cardiac fibrosis have been a hot issue in MI therapy.

Many studies have explored the amphoterininduced gene and open reading frame (AMIGO2), also known as Alivin-1; it is a novel member of the genes encoding for type I transmembrane proteins. AMIGO2 possesses a protein structure comprising an extracellular domain containing six leucine-rich repeats (LRRs) followed by an immunoglobulin domain, a transmembrane and intracellular domain with phosphorylation sites. The homo- and heterodimers lead to signal transduction $[5,6]$. Interestingly, AMIGO2 has been studied in cancer research, and its anti-apoptosis and migration abili-

Address for correspondence: Dr. Tianfu Fang, Department of Cardiology, Xixi Hospital of Hangzhou, Hengbu Street 2, Xihu District, Hangzhou, Zhejiang, China, tel:+86-021-63240090, fax:+86-021-63240091, e-mail: TianfuFang123@126.com 
ties of have been reported in many articles. The AMIGO2 deficiency in gastric adenocarcinoma cells changed the morphology, chromosomal stability and cell migration in nude mice [7]. Moreover, AMIGO2 has also been detected in proliferating synovial tissue invading cartilage and is found in rheumatoid arthritis patients [8].

It is well known that the phosphoinositide 3-kinase dependent kinase 1 (PDK1)-Akt pathway plays a vital role in multiple aspects of biological processes including cell growth, survival metabolism and angiogenesis. Dysregulation of this pathway is correlated with the pathogenesis of many diseases including cancer and metabolic, cardiovascular and neurological disorders $[9,10]$. As regulators of this pathway, PDK1 and Pten are commonly detected, and the Pten/Akt pathway is involved in inflammatory responses, apoptosis and angiogenesis, which play essential roles in the biological processes of cardiovascular cells [11, 12]. Pten has a molecular weight of $40-50 \mathrm{kDa}$ and comprises an $\mathrm{N}$-terminal $\mathrm{PIP}_{2}$-binding polybasic tail, an $\mathrm{N}$-terminal phosphatase domain, a C2 domain, and a C-terminal tail region with multiple phosphorylation sites $[13,14]$. Akt is at the central hub of the signaling pathway modulating multiple aspects of cellular functions including cellular apoptosis, proliferation, metabolism and hypertrophy [15, 16]. The activity of Akt is primarily controlled by Pten via the regulation of Ptdlns $[3,4,5] \mathrm{P}_{3}$ levels $[13,17]$. Furthermore, Hyojin Park et al. [18] have found that AMIGO2 directly interacts with PDK1, a primary activator of Akt at the plasma membrane in endothelial cells (ECs).

AMIGO2, as a key regulator, reduced apoptosis and stimulated proliferation via the Akt signalling pathway in cancer cells. In the field of cardiovascular research, there are hardly any reports on AMIGO2. The present aim is to observe the functional roles of AMIGO2 in ischemic heart disease and further investigate the underlying mechanism of the relationship among AMIGO2 and the Pten and Akt signaling pathways.

\section{Methods}

\section{Experimental animals}

All animal experiments were approved by the Animal Care and Use Committee of Xixi Hospital and followed the guidelines for the Care and Use of Laboratory Animals published by the National Institutes of Health. Wild-type (WT) C57BL/6 and AMIGO2 knockout (KO) mice (C57BL/6 background) were purchased from Beijing Vital
River Laboratory Animal Technology Company for research use only. Mice were fed in the cages at a temperature of $21 \pm 4^{\circ} \mathrm{C}$. The weight of mice suitable for MI surgery is $22-25 \mathrm{~g}$, and their age was $6-8$ weeks.

\section{Isolation of neonatal ventricular cardiomyocytes}

Neonatal ventricular cardiomyocytes (CMs) were isolated as described previously. Briefly, hearts from 24-h-old AMIGO2 KO and WT mice were excised and digested with $0.1 \%$ trypsin (Gibco, NY, USA). The dispersed cells were cultured with $10 \%$ FBS-supplemented high glucose DMEM (glucose concentration, $4 \mathrm{~g} / \mathrm{L}$ ) for $90 \mathrm{~min}$ at $37^{\circ} \mathrm{C}$ with $5 \% \mathrm{CO}_{2}$. Then, non-adherent cells were transferred into the bottom of 24- or 6-well plates (Corning, MA, USA) and cultured for $24 \mathrm{~h}$. Then, the CMs beat rhythmically and were well prepared for the follow-up experiments.

\section{Isolation of endothelial cells}

The murine ECs were isolated by the following procedures. Mice were sacrificed by an overdose of isoflurane, and the lungs were separated and minced into small pieces, washed in Hank's buffer and digested with dispase for $1 \mathrm{~h}$. The homogenate underwent filtration and centrifugation at $300 \mathrm{~g}$ for $5 \mathrm{~min}$. The cells were suspended and purified with anti-mouse VE-cadherin antibody-coated (Pharmingen, Germany) magnetic beads. The collected cells were cultured in DMEM (Invitrogen, Germany) supplemented with 20\% fetal calf serum, endothelial cell growth factor (Sigma Aldrich, USA), penicillin $(50 \mathrm{U} / \mathrm{mL}$ ) and streptomycin $(50 \mu \mathrm{g} / \mathrm{mL})$. The ECs from the first two passages were over $95 \%$ Dil-Ac-LDL positive for purification. Then, cells were prepared in passage 3-6 for the in vitro study.

\section{In vitro proliferation test}

The proliferation was tested by Ki67 immunostaining. Neonatal CMs were seeded at $1 \times 10^{4}$ cells per well in 24-well plates and cultured under normoxic conditions for 7 days. The proliferating cells were determined by double staining of Ki67 and DAPI. In brief, the cells were fixed in $4 \%$ paraformaldehyde, blocked with $5 \%$ bull serum albumin (BSA) after 10 min of permeabilization, and incubated with primary antibodies (Ki67, Abcam, USA) overnight followed by the respective fluorescent secondary antibodies. After three washes with PBST, the cell nuclei were stained with Hoechst 33258 pentahydrate $1 \mu \mathrm{g} / \mathrm{mL}$ (Invitrogen, USA). 
The fluorescence images were obtained at 5 random visual fields per well by a Leica fluorescence microscope. The proliferation rate is the number of Ki67/DAPI double positive cells divided by the total number of cells, and the data are presented as the mean \pm standard deviation (SD).

\section{In vitro apoptosis measurement}

The apoptotic CMs were detected by the terminal deoxynucleotidyl transferase-mediated dUPT nick end-labelling (TUNEL) assay (In Situ Cell Death Kit, TMR red, Roche Applied Science, USA). Neonatal CMs were seeded at $1 \times 10^{4}$ cells per well in 24-well plates and induced to apoptosis under hypoxic conditions $\left(0.1 \% \mathrm{O}_{2}, 5 \% \mathrm{CO}_{2}\right)$ in an FBS-free medium for $48 \mathrm{~h}$. In brief, the cells were fixed in $4 \%$ paraformaldehyde, permeabilized in $0.2 \%$ Triton X-100 and blocked with 5\% BSA. After 3 washes with $0.1 \%$ Tween-20 PBS, CMs were stained following manufacturer instructions. The cell nuclei were stained with Hoechst 33258 pentahydrate $1 \mu \mathrm{g} / \mathrm{mL}$ (Invitrogen, USA). Fluorescence photographs were taken at 5 random visual fields per well using a Leica fluorescence microscope. The apoptosis rate is the number of TUNEL/DAPI double-positive cells divided by the total number of cells, and the data are presented as the mean $\pm \mathrm{SD}$.

\section{Tube formation evaluation}

To investigate the angiogenesis potential of ECs in vitro, cells were cultured at a concentration of $2 \times 10^{4}$ cells onto each growth factor-reduced Matrigel-coated (BD Pharmingen, CA, USA) well of a 96 -well plate. After $4-6 \mathrm{~h}$ of incubation, the capillary network structures of the ECs were photographed using phase-contrast microscopy (Leica, Wetzlar, Germany), and the total number of branches were calculated by Image-Pro software. The data are presented as the mean $\pm \mathrm{SD}$.

\section{Mouse MI model}

Mice were used for the MI model by ligation of the left anterior descending coronary artery (LAD) $[19,20]$. In brief, mice were anesthetized with an intraperitoneal injection of $4 \%$ chloral hydrate $(4 \mathrm{mg} / \mathrm{kg}$ ) and ventilated, and a left thoracotomy was created. The LAD was ligated permanently with an 8-0 silk suture. Successful coronary occlusion was verified by the observed myocardium blanching distal to the ligation. Paleness around and below the ligation point indicated a successful operation. The chest was closed, and the mice were placed back into cages.

\section{Cardiac function assessment} by echocardiography and hemodynamics

The mice underwent echocardiography at 3 days and 28 days post-MI, whereas a hemodynamics evaluation was implemented 28 days post-MI. In brief, the mice were anesthetized via the inhalation of $2 \%$ isoflurane in $100 \% \mathrm{O}_{2}$ gas; the 2-dimensional and $\mathrm{M}$-model echocardiographic images $(17.5 \mathrm{MHz}$ transducer, Vevo 2100, Visual Sonics, Canada) were obtained at the level of the papillary muscles. The left ventricular end-systolic diameter (LVESD) and left ventricular end-diastolic diameter (LVEDD) were measured during at least 3 separate cardiac cycles. The ejection fraction $(\mathrm{EF}, \%)$ and fractional shortening (FS, \%) were calculated by the Simpson method.

Cardiac catheterization was performed with a catheter conducer (Millar Instrument, Houston, TX, USA) for hemodynamic assessment. A $1.4 \mathrm{~F}$ pressure catheter, SPR 671, was inserted into the aorta and left ventricle (LV) through the right carotid artery. The transducer was tested to measure the left ventricular systolic pressure (LVSP), left ventricular end diastolic pressure (LVEDP), and $\mathrm{LV}$ maximum $\pm \mathrm{dp} / \mathrm{dt}$.

All measurements were repeated three times by a blinded investigator, and the data are presented as the mean $\pm \mathrm{SD}$.

\section{Histology analysis}

The animals were sacrificed, and the isolated hearts were immediately placed in a $10 \% \mathrm{KCl}$ solution to induce the hearts to remain in the diastolic phase. The left and right atria of the heart were removed, leaving the $\mathrm{LV}$, and dehydrated in a $30 \%$ sucrose solution for $12-24 \mathrm{~h}$ at $4^{\circ} \mathrm{C}$. The samples were embedded in Tissue-Tek OCT (Sakura Finetek, CA, USA) compound and snap frozen in liquid nitrogen. The frozen sections were cut to a $7-\mu \mathrm{m}$ thickness.

The 3-days-post-MI samples were incubated with primary antibodies (TnI, Abcam, USA) overnight followed by the fluorescent conjugated secondary antibodies. After 3 washes with PBST, the apoptotic CMs were stained by a TUNEL kit. The cell nuclei were stained with Hoechst 33258 pentahydrate $1 \mu \mathrm{g} / \mathrm{mL}$ (Invitrogen, USA). Fluorescence photographs were obtained with a Leica fluorescence microscope. The ratio of TUNEL-positive CMs was measured using the quantitative software Image-Pro Plus. The apoptosis rate is the number of TUNEL/DAPI double positive cells divided by the total number of cells, and the data are presented as the mean $\pm \mathrm{SD}$. 
Table 1. Primer Sequence of mRNA for RT-PCR.

\begin{tabular}{|c|c|c|c|}
\hline Gene name & Primer sequence & Gene ID & Product size \\
\hline Mus-AMIGO-2 F & GGAGGTTCAAGCTGGCTGAT & NM 001164602.1 & 620 \\
\hline Mus-AMIGO-2 R & GTGGGCGTGGGATCTGTTTA & & \\
\hline Mus-PDK-1 F & TGTCTACGGTGGAAACCAGC & XM 01731725.1 & 337 \\
\hline Mus-PDK-1 R & TTGGCTTCTGGTCGGAGTTC & & \\
\hline Mus-Pten F & GAAAGGGACGGACTGGTGTA & NM 008960.2 & 213 \\
\hline Mus-Pten R & TCTTGTGAAACAGCAGTGCC & & \\
\hline Mus-Akt F & CTGCCCTTCTACAACCAGGA & XM 006501107.1 & 239 \\
\hline Mus-Akt R & САTACACATCCTGCCACACG & & \\
\hline Mus-Bcl-2 F & TTGTAATTCATCTGCCGCCG & NM 009741.5 & 179 \\
\hline Mus-Bcl-2 R & AATGAATCGGGAGTTGGGGT & & \\
\hline Mus-Bax F & TCATGAAGACAGGGGCCTTT & NM 007527.3 & 197 \\
\hline Mus-Bax R & GTCCACGTCAGCAATCATCC & & \\
\hline Mus-Casp-3 F & CAGCCAACCTCAGAGAGACA & NM 009810.3 & 190 \\
\hline Mus-Casp-3 R & ACAGGCCCATTTGTCCCATA & & \\
\hline Mus-VEGF-F & CAAACCTCACCAAAGCCAGC & NM 001110267.1 & 187 \\
\hline Mus-VEGF-R & ACGCGAGTCTGTGTTTTTGC & & \\
\hline Mus-Actin F & GTGGGAATGGGTCAGAAGGA & NM 007393.5 & 226 \\
\hline Mus-Actin R & TCATCTTTTCACGGTTGGCC & & \\
\hline
\end{tabular}

RT-PCR — real-time polymerase chain reaction

The 7-days-post-MI heart samples were incubated with the primary antibodies Ki67 and $\mathrm{TnI}$ (Abcam, USA) diluted 1:200 at $4^{\circ} \mathrm{C}$ overnight, followed by incubation of secondary fluorescent antibodies. The nuclei were stained with Hoechst 33258 (Thermo Fisher Scientific, CA, USA), and the images were observed by a fluorescence microscope (Leica, Wetzlar, Germany). The proportion of Ki67-positive CMs was analyzed using the quantitative software Image-Pro Plus. The proliferation rate is the number of Ki67/DAPI double positive cells divided by the total number of cells, and the data were are presented the mean $\pm \mathrm{SD}$.

The heart samples were incubated with primary antibody reacted (Abcam, USA) with $\alpha$-SMA, CD31 and primary antibody with troponin I (TnI) (Abcam, USA) diluted 1:200 at $4^{\circ} \mathrm{C}$ overnight, followed by incubation of the secondary fluorescent antibodies. The nuclei were stained with Hoechst 33258 (Thermo Fisher Scientific, CA, USA), and images were taken with a fluorescence microscope (Leica, Wetzlar, Germany). The number of $\alpha$-SMA or CD31 positive vessels were analysed using the quantitative software Image-Pro Plus. The data are presented as the mean \pm SD.

The scar size of the 28-day-post-MI samples was measured by the Masson-trichrome method. Briefly, frozen tissue sections of heart tissues from different groups were fixed in $4 \%$ paraformaldehyde and stained with the Masson-trichrome kit. The total LV area was measured using Image-Pro Plus. The infarct area was calculated as the percentage $(\%)$ of the infarcted area divided by the entire LV. The data are presented as the mean \pm SD.

\section{Quantitative real-time PCR}

Total RNA was extracted from the CMs using Trizol reagent (Invitrogen, CA, USA) according to manufacturer instructions. The cDNA was synthesized from $2 \mu \mathrm{g}$ of RNA using Moloney Murine Leukemia Virus (M-MLV) reverse transcriptase and an oligo dT 18 primer (TAKARA, Japan). Quantitative real-time polymerase chain reaction (PCR) was performed using the SYBR Green Reaction Mix (TAKARA, Japan) following manufacturer instructions. The PCR conditions were $95^{\circ} \mathrm{C}$ for $10 \mathrm{~min}$ and 40 cycles of $95^{\circ} \mathrm{C}$ for $30 \mathrm{~s}, 60^{\circ} \mathrm{C}$ for $30 \mathrm{~s}$ and $72^{\circ} \mathrm{C}$ for $1 \mathrm{~min}$. The PCR primers were designed using Primer3 Input online software, and they are listed in Table 1. Beta-actin was used as a control, and the relative expression of the target genes was evaluated by a comparative cycle threshold method and normalized to the control. The data are presented as the mean $\pm \mathrm{SD}$. 


\section{Western blot analysis}

After $48 \mathrm{~h}$ of incubation under hypoxic conditions and FBS-free stimulation, the CMs and ECs were lysed with a lysis buffer ( $50 \mathrm{mM}$ HEPE, $5 \mathrm{mM}$ EDTA, and $50 \mathrm{mM} \mathrm{NaCl}$, Beyotime Biotechnology, China) and protease inhibitor (Roche Applied Science, IN, USA) for $30 \mathrm{~min}$ and centrifuged at $12,000 \mathrm{rpm}$ for $30 \mathrm{~min}$ at $4^{\circ} \mathrm{C}$ to obtain extracts. The protein concentrations were detected by a BCA Protein Assay Kit (Life Technologies, USA), and the samples $(30 \mu \mathrm{g})$ were electrophoresed on SDS-polyacrylamide gel. After the protein transfer, the PVDF membranes were blocked with PBS-0.05\% Tween (PBST) containing $5 \% \mathrm{BSA}$ and reacted with primary antibodies including anti-AMIGO2 (1:500, Abcam, USA), Pten (1:500, CST, USA), Bcl-2 (1:500, CST, USA), Bax (1:500, CST, USA), caspase-3 (1:500, CST, USA), NF $\kappa \mathrm{B} /$ /p56 (1:500, CST, USA), p-Akt, T-Akt (1:500, CST, USA), VEGF (1:500, Abcam, USA) and $\beta$-actin (Abcam, USA) overnight at $4^{\circ} \mathrm{C}$. After two washes with PBST, the membranes were incubated with 1:2000 secondary antibodies conjugated with HRP antibody at room temperature for $1 \mathrm{~h}$. The membranes were visualized by a chemiluminescence system (BioRad, USA). $\beta$-actin was used as the control for evaluating the relative expression of other proteins.

\section{Statistical analysis}

All of the data are presented as the mean \pm SD. Statistical analyses for the measurement of significant differences between the AMGIO2 KO and WT groups were performed with the Student t-test. Probability values of $\mathrm{p}<0.05$ were considered statistically significant.

\section{Results}

The lack of AMIGO2 results in higher apoptosis ratio in vitro

To examine the biological role of AMIGO2 in $\mathrm{CMs}$ apoptosis, the CMs isolated from AMIGO2 and WT mice underwent a 24-h hypoxia induction. TUNEL-positive cells were evaluated and it was found that the AMIGO2 deficiency led to the increased apoptosis of myocytes when compared with the WT group (Fig. 1A, D, \#p < 0.05).

\section{Deletion of AMIGO2 results in lower $\mathrm{CM}$ proliferation in vitro}

To determine the functional role of AMIGO2 in $\mathrm{CM}$ proliferation, Ki67 immunostaining was performed. The Ki67-positive cells were counted and it was found that AMIGO2-deficient hearts had fewer proliferative CMs than the WT cells (Fig. 1B, E, \# p 0.05).

\section{Absence of AMIGO2 induces weaker proliferation ability of endothelial cells in vitro}

To investigate the functional role of AMIGO2 in endothelial cell proliferation, tube formation was performed. The length of the capillary structure in the AMIGO2 KO and WT groups was calculated and it was found that AMIGO2-deficient endothelial cells had a weaker ability of microvessel formation than the WT hearts (Fig. 1C, F, \#p < 0.05).

\section{The absence of AMIGO2 suppresses} proliferation and induces apoptosis in vivo

The role of AMIGO2 in apoptosis in vivo was validated by TUNEL staining in 3-day samples. Findings suggest that the deficiency of AMIGO2 markedly induced more apoptotic nuclei in the border zone compared with WT group. The proliferation ratio of the myocytes in the border area of 7-day samples was measured by immunostaining with Ki67 antibody. Fewer Ki67-positive CMs in AMIGO2 KO border zones were observed (Fig. 2A-D, \#p < 0.05).

\section{The suppression of AMIGO2 restrains angiogenesis in vivo}

The role of AMIGO2 in angiogenesis in vivo using $\alpha$-SMA/CD31 immunofluorescence staining was validated. Findings suggest that the deficiency of AMIGO2 markedly restrained microvessel formation in the border zone compared with the WT group (Fig. 2E-H, \#p < 0.05).

\section{Deficiency of AMIGO2 deteriorates cardiac function and neovascularization and increases infarct size in vivo}

To determine whether AMIGO2 plays a key role in cardiac function, the myocardial contractile parameters at 3 and 28 days after MI were evaluated. The echocardiograph results indicated AMIGO2 KO induced weaker heart function compared with the WT group (Fig. 3A-D), including a worse EF and FS (Fig. 3A, B, \#p < 0.05). Furthermore, the LVED of both the end-diastolic and end-systolic stages were shorter in the AMIGO2 KO mouse hearts than in the WT group (Fig. 3C, D, \#p < 0.05). Moreover, the hemodynamic results showed a similar effect. Compared with the WT group, AMIGO2 KO mice had a significantly lower LVSP, higher LVEDP, and lower \pm dp/dt (Fig. 3E-H, \#p < 0.05). 


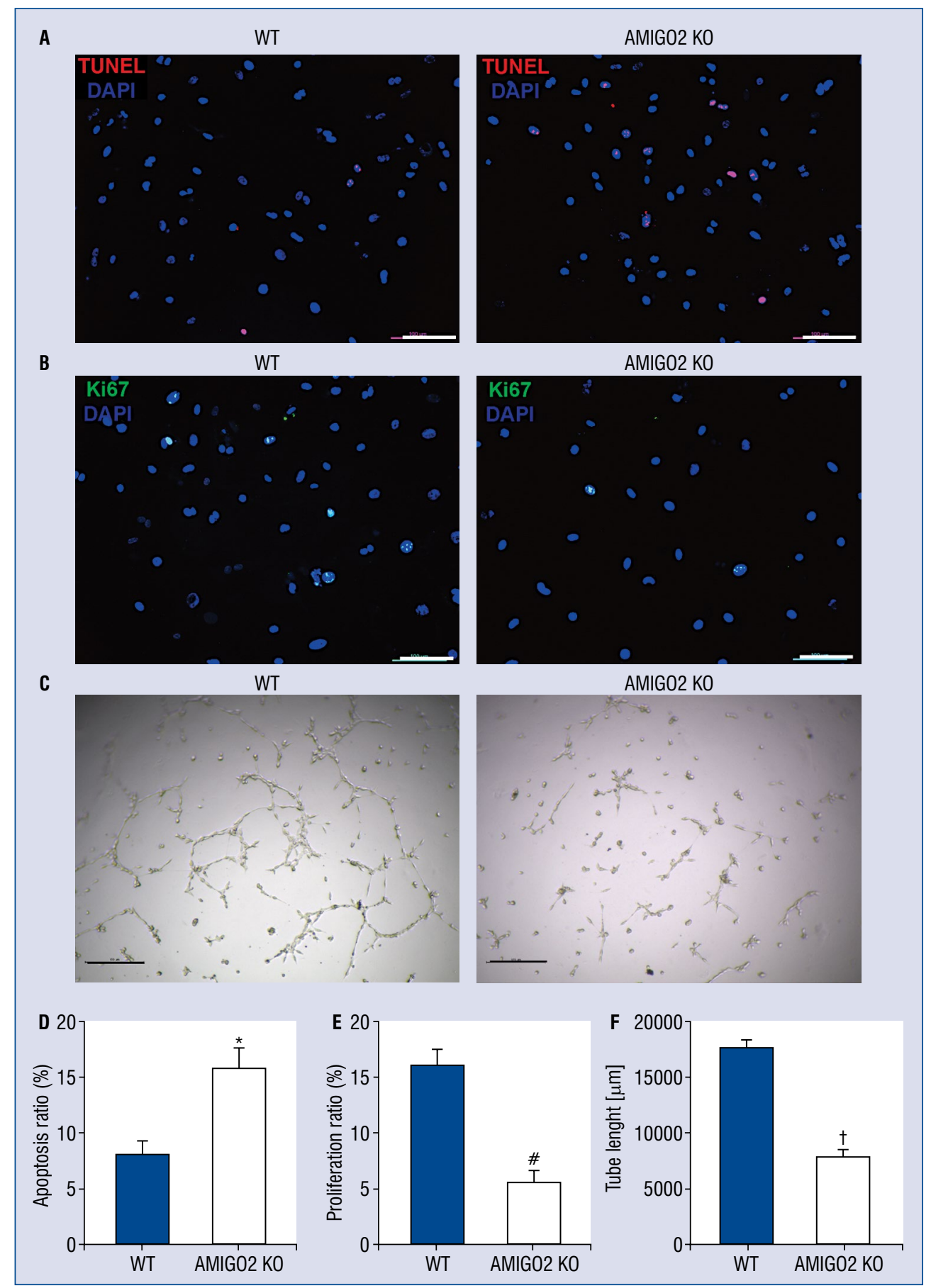

Figure 1. Suppression of AMIGO2 results in decreased apoptosis and increased proliferation of cardiomyocytes (CMs) and stimulates angiogenesis of endothelial cells (ECs) in vitro; A. Representative pictures of TUNEL-positive CMs. Scale bar: $100 \mu \mathrm{m}$; B. Representative pictures of Ki67-positive CMs. Scale bar: $100 \mu \mathrm{m}$; C. Representative pictures of tube formation of ECs. Scale bar: $100 \mu \mathrm{m}$; D. Quantification of the apoptotic CMs (n = 5/group); ${ }^{*} p<0.01 \mathrm{vs}$. the Wild-type (WT) group; E. Quantification of the proliferative CMs ( $n=5 /$ group); \#p < 0.01 vs. the WT group; F. Quantitative analysis of tube length ( $n=5$ /group); ${ }^{\dagger} p<0.01$ vs. other groups.

To evaluate the effect of AMIGO2 in angiogenesis and infarct size, $\alpha$-SMA/CD31 immunostaining and Masson's trichrome staining were used. The quantitative assessment of myocardial fibrosis indicated that AMIGO2-deficient mice had more obvious neovascularization and a smaller scar area compared with the WT group (Fig. 4A, B, $\# \mathrm{p}<0.05)$. 


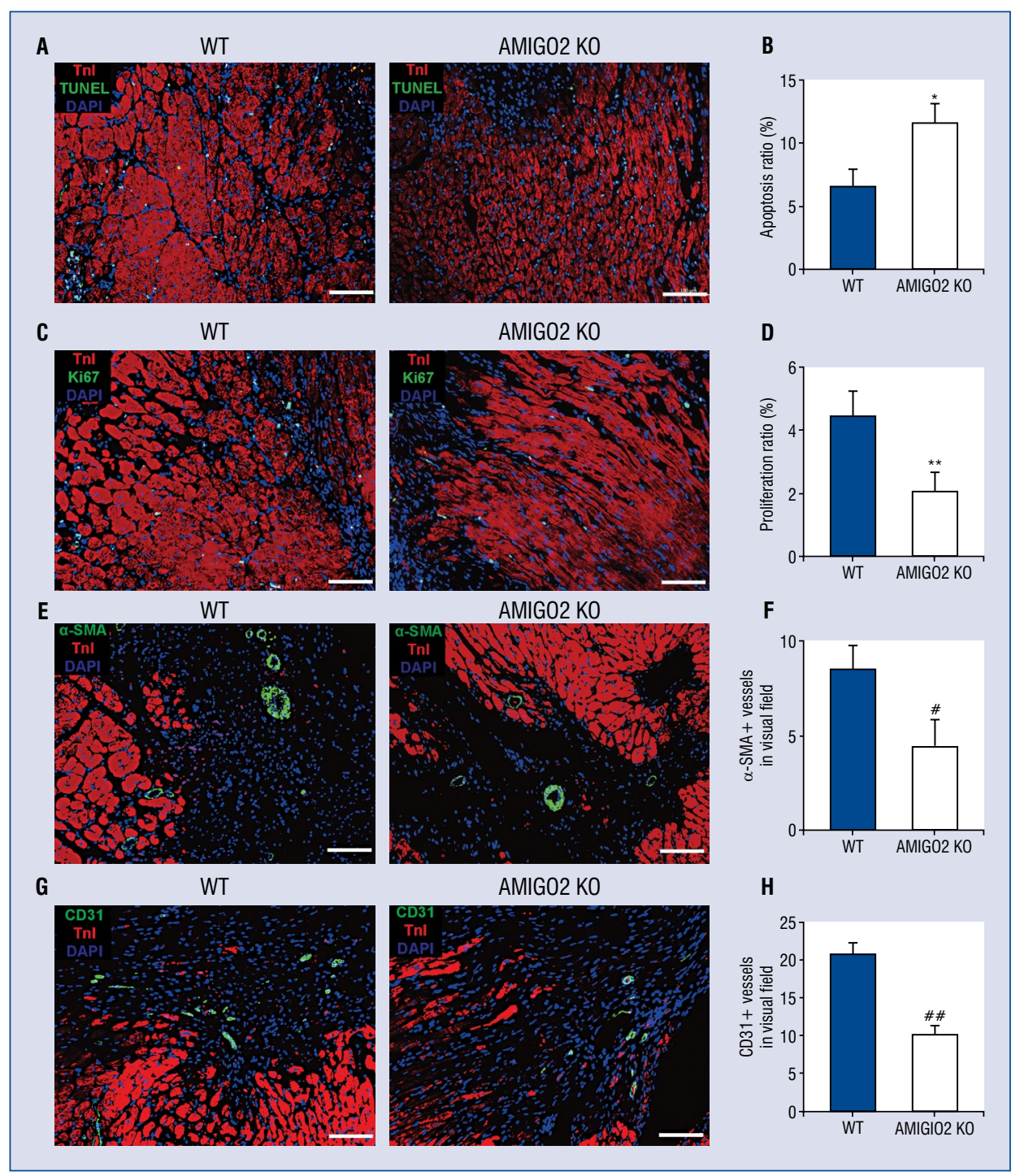

Figure 2. The lack of AMIGO2 reduced apoptosis, induced proliferation and increased neovasculation in vivo; $\mathbf{A}$. Representative pictures of TUNEL-positive nuclei cardiomyocytes (CMs) in border zone. Scale bar: $100 \mu \mathrm{m}$; B. Quantification of the apoptotic nuclei ( $n=5 /$ group). ${ }^{*} p<0.01$ vs. the Wild-type (WT) group; C. Representative pictures of Ki67-positive CMs in border zone. Scale bar: $100 \mu \mathrm{m}$; D. Quantification of the proliferative CMs ( $=5 /$ group); ${ }^{* *} p<0.01$ vs. the WT group; E. Representative pictures of $\alpha$-SMA-positive small arteries in border zone. Scale bar: $100 \mu \mathrm{m}$; F. Quantification of $\alpha$-SMA-positive small arteries ( $\mathrm{n}=5 / \mathrm{group}$ ); $\# \mathrm{p}<0.05$ vs. other groups; G. Representative pictures of CD31-positive microvessels in border zone. Scale bar: $100 \mu \mathrm{m}$; H. Quantification of CD31-positive microvessels ( $\mathrm{n}=5$ /group); ${ }^{\# \#} \mathrm{p}<0.05$ vs. other groups.

\section{AMIGO2 deficiency influences both} the mRNAs and proteins of the PDK-1 and Akt signalling pathways in myocytes

To explore the underlying mechanism of anti-apoptosis during AMIGO2 loss in CMs, the relative expression of proteins by RT-PCR and immunoblotting were analyzed. Results showed that a deficiency of AMIGO2 stimulates a different expression of PDK-1 and Akt signalling pathway molecules at mRNA and protein levels (Fig. 4C-H, $\# \mathrm{p}<0.05)$.

\section{Discussion}

The mechanism of ischemic heart disease still requires intensive study. In the present study, assessed first was the role of AMIGO2 in inhibiting $\mathrm{CM}$ loss and stimulating the proliferation of sur- 


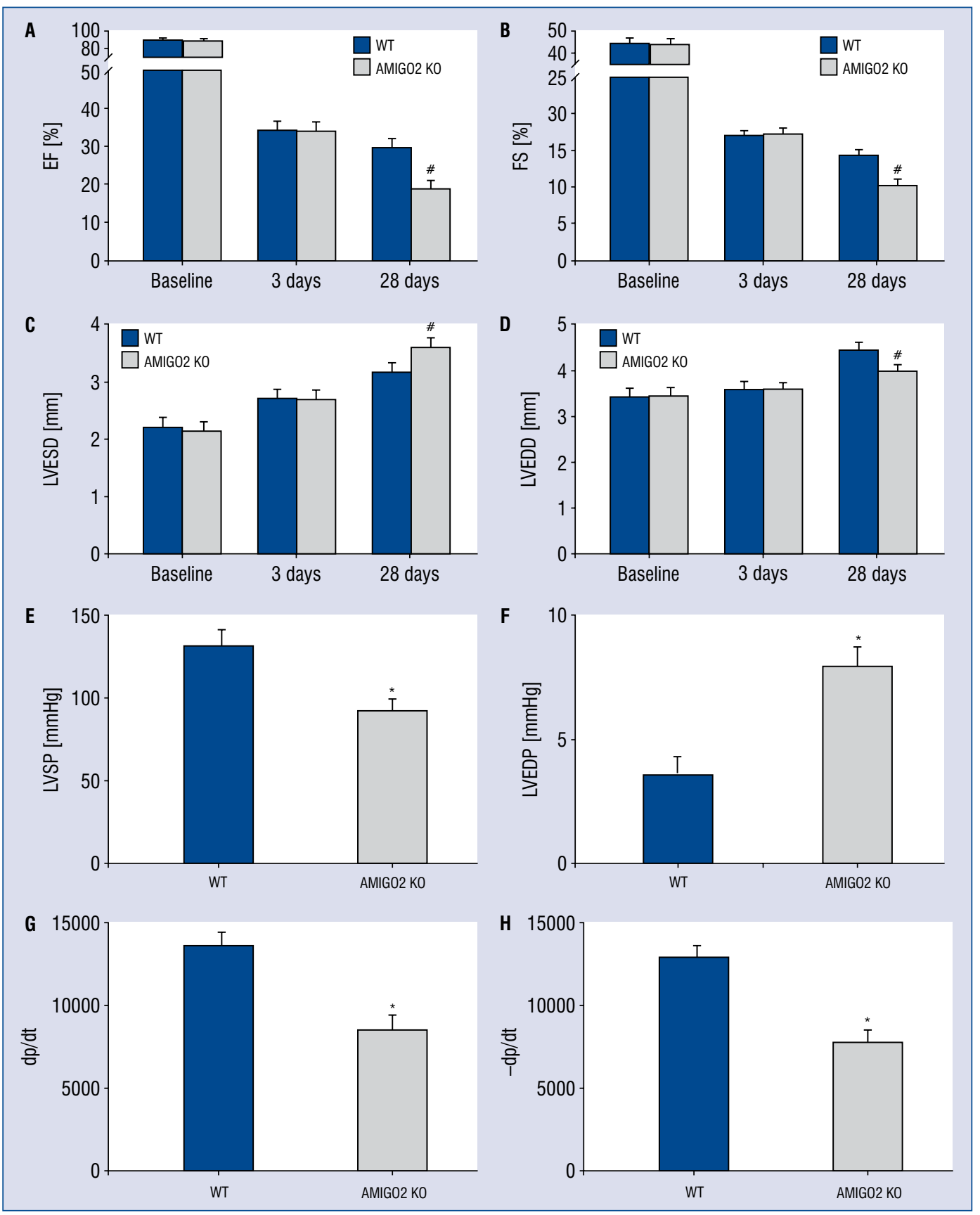

Figure 3. Deletion of AMIGO2 benefits cardiac function recovery; A. Quantitative analysis of echocardiography; A-D. Quantitative analysis of echocardiography; \#p < 0.01 vs. the Wild-type (WT) group; E-H. Quantitative analysis of hemodynamics; * $\mathrm{p}<0.01$ vs. the WT group; other abbreviations - see text.

vival cardiac cells in the infarcted heart. The main findings are listed as follows: (1) lack of AMIGO2 induces the damages of ischemic heart disease; (2) the absence of AMIGO2 leads to more myocardium apoptosis and less $\mathrm{CM}$ and EC proliferation in both in vitro and in vivo experiments; and (3) AMIGO2 deficiency causes variable expression of the PDK1, Pten, Bcl-2/Bax and Akt signalling pathways. The data first revealed the role of AMIGO2 in ischemic heart disease; AMIGO2 deficiency ameliorates damage to infarcted heart and cardiovascular cells by activating the PDK- 1 and Akt signalling pathway. Furthermore, according to the present results, AMIGO2 may be a potential target manipulating ischemic heart injury. Synthetic up-regulation may enhance myocardium salvage and neovasculariza- 


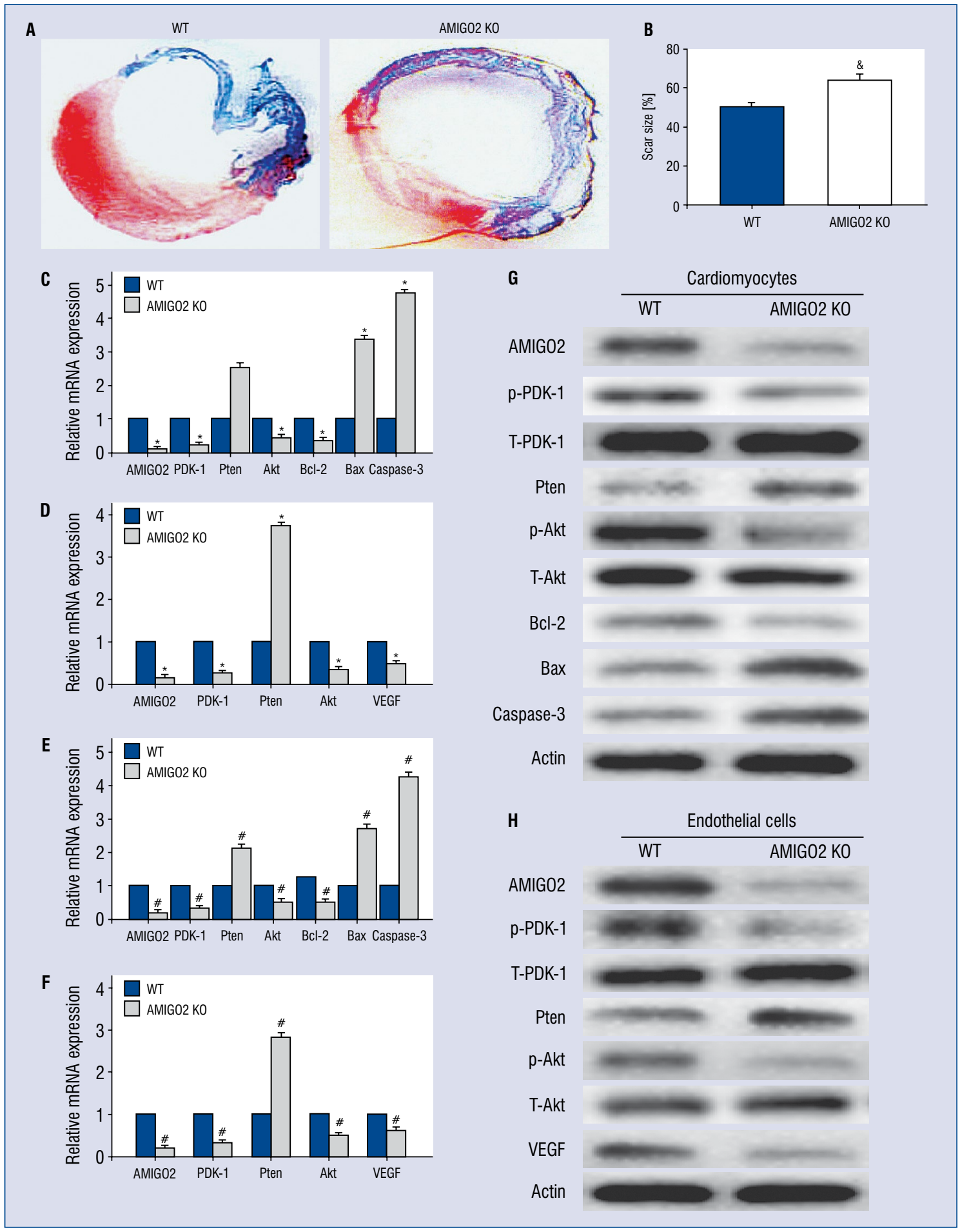

Figure 4. Deficient AMIGO2 decreased infarct size, activated the PDK-1, Pten, Akt, Bcl-2/Bax and VEGF signalling pathways in cardiomyocytes (CMs) and endothelial cells (ECs) at the levels of the mRNAs and protein; A. Representative Masson's trichrome staining of the heart to show the infarct zone 28 days after myocardial infarction; B. Quantification of infarct zone in heart tissue; ${ }^{\&} p<0.01$ vs. the Wild-type (WT) group; C, D. Quantitative analysis of mRNA expression of PDK-1, Pten, Akt, Bcl-2/Bax, caspase-3 and VEGF; \#p < 0.01 vs. the WT group; E, F. Quantitation of PDK-1, Pten, Akt, Bcl-2/Bax, caspase-3 and VEGF expression in CMs and ECs, $n=3 ; \# p<0.01$ vs. the WT group; G. H. Western blotting identification for altered expression of NF- $\kappa$ B/p56 Akt, Bcl-2/Bax, caspase-3 and VEGF. 
tion, which accelerate recovery from infarction damage. Additionally, PDK-1 may be another key point of the downstream pathway of AMIGO2. The abolishment of PDK-1 could terminate the ischemic injury induced by AMIGO2 deficiency.

Apoptosis is a suicidal mechanism regulated by a conserved genetic program [21]. Mitochondria are a major organelle participating in the apoptosis [22]. Once the apoptotic signal is transferred into the mitochondria, the stability of the mitochondrial membrane is vulnerable. Proteins in the $\mathrm{Bcl}-2$ and caspase family play substantial roles in apoptosis, modulating signal transduction and undergoing these processes. Bcl-2 inhibits apoptosis by heterodimer formation, whereas Bax functions reverse to that as a pro-apoptotic protein [23]. Bcl-2 and Bax both participate in the apoptosis of CMs, and they are essential factors in myocytes under ischemic conditions [24, 25]. Caspase proteins and $\mathrm{Bcl}-2 / \mathrm{Bax}$ are associated with apoptosis. They play critical roles in cardioprotection while PDK1, Pten and Akt are their powerful upstream regulators [26, 27]. The present data indicates that AMIGO2 is related to the caspase family and $\mathrm{Bcl}-2$, suggesting that $\mathrm{AMIGO} 2$ is involved in myocardial apoptosis.

Recently, several studies have reported the biological functions of AMIGO2 in different types of cells. Among the many biological properties of AMIGO2, apoptosis induction and proliferation inhibition have gained the most attention of researchers. In the field of oncology, a higher level of AMIGO2 protein was proven to be closely relevant to the growth and development of cancer. In gastric cancer cells, highly expressed AMIGO2 in gastric cancer cells mediated more apoptosis and lower proliferation via the Wnt and Hedgehog pathway. Although the effect of AMIGO2 in cancer research has been studied, the functional role of AMIGO2 in the field of cardiovascular research remains unknown. The apoptosis and proliferation of the myocardium was first measured, both in vitro and in vivo, in an AMIGO2-deficient MI mouse model. Moreover, the heart function and hemodynamics of the AMIGO2 KO mice was detected and the related pathway for these phenotype changes was measured. The present results provided strong evidence for the apoptosis-resistant role of AMIGO2 in CMs both in vitro and in vivo. In this study, we found decreased Bcl-2 expression and increased Bax expression caused by AMIGO2 deficiency in CMs. The data demonstrated that the absence of AMIGO2 induced $\mathrm{Bcl}-2 / \mathrm{Bax}$ down-regulation to aggravate damage to cardiovascular function from ischemic injury.

\section{Limitations of the study}

However, the present study also has some limitations. First, the interaction between AMIGO2 and PDK1 has not been proven. According to the achievement of Hyojin Park et al. [18], the direct binding of AMIGO2 to the PH domain of PDK1 enhanced the activation of PDK1 and Akt, and the PTD-A2, a specific peptide in the $\mathrm{C}$-terminal region of AMIGO2, effectively abrogated the phosphorylation of PDK1 and Akt activities. The effect of PTDA2 in cardiovascular cells should be confirmed. Second, the role of PDK1 as an upstream regulator of the Akt pathway in cardiovascular cells needs to be proven. The suppression or overexpression of PKD1 by lenti-viral transfection is required for phenotype and pathway validation. Last, without ischemic injury, the effect of AMIGO2 in cardiovascular cells has not been confirmed in this article. The work herein demonstrated that AMIGO2 deficiency induced greater damage to the heart in the MI model; however, the role of AMIGO2 deletion in normal hearts should be investigated.

\section{Conclusions}

In conclusion, the deletion of AMIGO2 resulted in a significantly higher apoptosis ratio and lower proliferation in cardiovascular cells after ischemic damage. The impairment caused by AMIGO2 deficiency was associated with the various expressions of PDK1, Pten, Akt, VEGF, $\mathrm{Bcl}-2 / \mathrm{Bax}$ caspase proteins. These data suggest that AMIGO2 may be another novel target for MI treatment.

\section{Conflict of interest: None declared}

\section{References}

1. Alpert JS, Thygesen K, Antman E, et al. Myocardial infarction redefined--a consensus document of The Joint European Society of Cardiology/American College of Cardiology Committee for the redefinition of myocardial infarction. J Am Coll Cardiol. 2000; 36(3): 959-69.

2. Kitsis RN, Peng CF, Cuervo AM. Eat your heart out. Nat Med. 2007; 13(5): 539-541, doi: 10.1038/nm0507-539, indexed in Pubmed: 17479097.

3. White HD, Norris RM, Brown MA, et al. Left ventricular endsystolic volume as the major determinant of survival after recovery from myocardial infarction. Circulation. 1987; 76(1): 44-51, indexed in Pubmed: 3594774.

4. Pluta A, Stróżecki P, Krintus M, et al. Left ventricular remodeling and arterial remodeling in patients with chronic kidney disease stage 1-3. Ren Fail. 2015; 37(7): 1105-1110, doi: 10.310 9/0886022X.2015.1061669, indexed in Pubmed: 26156686. 
5. Kuja-Panula J, Kiiltomäki M, Yamashiro T, et al. AMIGO, a transmembrane protein implicated in axon tract development, defines a novel protein family with leucine-rich repeats. J Cell Biol. 2003; 160(6): 963-973, doi: 10.1083/jcb.200209074, indexed in Pubmed: 12629050.

6. Kajander T, Kuja-Panula J, Rauvala H, et al. Crystal structure and role of glycans and dimerization in folding of neuronal leucine-rich repeat protein AMIGO-1. J Mol Biol. 2011; 413(5): 1001-1015, doi: 10.1016/j.jmb.2011.09.032, indexed in Pubmed: 21983541.

7. Rabenau KE, O'Toole JM, Bassi R, et al. DEGA/AMIGO-2, a leucine-rich repeat family member, differentially expressed in human gastric adenocarcinoma: effects on ploidy, chromosomal stability, cell adhesion/migration and tumorigenicity. Oncogene. 2004; 23(29): 5056-5067, doi: 10.1038/sj.onc.1207681, indexed in Pubmed: 15107827.

8. Andersson U, Harris HE. The role of HMGB1 in the pathogenesis of rheumatic disease. Biochim Biophys Acta. 2010; 1799(1-2): 141-148, doi: 10.1016/j.bbagrm.2009.11.003, indexed in Pubmed: 20123076.

9. Thompson CB. Apoptosis in the pathogenesis and treatment of disease. Science. 1995; 267(5203): 1456-1462.

10. Dimmeler S, Zeiher AM. Endothelial cell apoptosis in angiogenesis and vessel regression. Circ Res. 2000; 87(6): 434-439, indexed in Pubmed: 10988233.

11. Sheppard K, Kinross KM, Solomon B, et al. Targeting PI3 kinase/ AKT/mTOR signaling in cancer. Crit Rev Oncog. 2012; 17(1): 69-95, indexed in Pubmed: 22471665.

12. Raimondi C, Falasca M. Targeting PDK1 in cancer. Curr Med Chem. 2011; 18(18): 2763-2769, indexed in Pubmed: 21568903.

13. Maehama T, Taylor GS, Dixon JE. PTEN and myotubularin: novel phosphoinositide phosphatases. Annu Rev Biochem. 2001; 70: 247-279, doi: 10.1146/annurev.biochem.70.1.247, indexed in Pubmed: 11395408.

14. Leslie NR, Downes CP. PTEN: The down side of PI 3-kinase signalling. Cell Signal. 2002; 14(4): 285-295, indexed in Pubmed: 11858936

15. Shiojima I, Walsh K. Regulation of cardiac growth and coronary angiogenesis by the Akt/PKB signaling pathway. Genes Dev. 2006; 20(24): 3347-3365, doi: 10.1101/gad.1492806, indexed in Pubmed: 17182864.

16. Vadlakonda L, Dash A, Pasupuleti M, et al. The Paradox of AktmTOR Interactions. Front Oncol. 2013; 3: 165, doi: 10.3389/ /fonc.2013.00165, indexed in Pubmed: 23802099.

17. Vanhaesebroeck B, Leevers SJ, Ahmadi K, et al. Synthesis and function of 3-phosphorylated inositol lipids. Annu Rev Biochem.
2001; 70: 535-602, doi: 10.1146/annurev.biochem.70.1.535, indexed in Pubmed: 11395417.

18. Park H, Lee S, Shrestha P, et al. AMIGO2, a novel membrane anchor of PDK1, controls cell survival and angiogenesis via Akt activation. J Cell Biol. 2015; 211(3): 619-637, doi: 10.1083/ /jcb.201503113, indexed in Pubmed: 26553931.

19. Xiao J, Moon M, Yan L, et al. Cellular FLICE-inhibitory protein protects against cardiac remodelling after myocardial infarction. Basic Res Cardiol. 2012; 107(1): 239, doi: 10.1007/s00395-0110239-z, indexed in Pubmed: 22202974.

20. Sun Y, Yi W, Yuan Y, et al. C1q/tumor necrosis factor-related protein-9, a novel adipocyte-derived cytokine, attenuates adverse remodeling in the ischemic mouse heart via protein kinase A activation. Circulation. 2013; 128(11 Suppl 1): S113-S120, doi: 10.1161/CIRCULATIONAHA.112.000010, indexed in Pubmed: 24030394.

21. Li J, Yuan J. Caspases in apoptosis and beyond. Oncogene. 2008; 27(48): 6194-6206, doi: 10.1038/onc.2008.297, indexed in Pubmed: 18931687.

22. Newmeyer DD, Ferguson-Miller S. Mitochondria: releasing power for life and unleashing the machineries of death. Cell. 2003; 112(4): 481-490, indexed in Pubmed: 12600312.

23. Cory S, Adams JM. The Bcl2 family: regulators of the cellular life-or-death switch. Nat Rev Cancer. 2002; 2(9): 647-656, doi: 10.1038/nrc883, indexed in Pubmed: 12209154.

24. Cook SA, Sugden PH, Clerk A. Regulation of bcl-2 family proteins during development and in response to oxidative stress in cardiac myocytes: association with changes in mitochondrial membrane potential. Circ Res. 1999; 85(10): 940-949, indexed in Pubmed: 10559141.

25. Misao J, Hayakawa Y, Ohno M, et al. Expression of bcl-2 protein, an inhibitor of apoptosis, and Bax, an accelerator of apoptosis, in ventricular myocytes of human hearts with myocardial infarction. Circulation. 1996; 94(7): 1506-1512, indexed in Pubmed: 8840837.

26. Li W, Wang H, Kuang CY, et al. An essential role for the Id1/ /PI3K/Akt/NFkB/survivin signalling pathway in promoting the proliferation of endothelial progenitor cells in vitro. Mol Cell Biochem. 2012; 363(1-2): 135-145, doi: 10.1007/s11010-011-1166-x, indexed in Pubmed: 22139302.

27. Hussain AR, Ahmed SO, Ahmed M, et al. Cross-talk between $\mathrm{NFkB}$ and the PI3-kinase/AKT pathway can be targeted in primary effusion lymphoma (PEL) cell lines for efficient apoptosis. PLoS One. 2012; 7(6): e39945, doi: 10.1371/journal. pone.0039945, indexed in Pubmed: 22768179. 